\title{
LETTER
}

\section{The harm of high-frequency oscillatory ventilation (HFOV) in ARDS is not related to a high baseline risk of acute cor pulmonale or short-term changes in hemodynamics}

\author{
Federico Angriman 1,2,3, Bruno L. Ferreyro 2,3,4, Lachlan Donaldson 1,2, Brian H. Cuthbertson 1,2,3, \\ Niall D. Ferguson ${ }^{2,3,4,5}$, Casper W. Bollen ${ }^{6}$, Thomas E. Bachman7, François Lamontagne ${ }^{8}$ \\ and Neill K. J. Adhikari ${ }^{1,2,3^{*}}$ (])
}

(c) 2019 Springer-Verlag GmbH Germany, part of Springer Nature

\section{Dear Editor,}

The acute respiratory distress syndrome (ARDS) may encompass sub-populations that respond differently to treatments [1]. High-frequency oscillatory ventilation (HFOV) related harm may be due to ventilator-induced lung injury (VILI) or hemodynamic instability explained by right ventricle (RV) failure that is present at baseline or that develops after HFOV is started [2]. Our objective was to evaluate whether the baseline risk of RV failure modified the effect of HFOV on mortality among ARDS patients and whether the effect of HFOV was mediated by short-term changes in hemodynamics.

We performed a secondary analysis of an individual patient data meta-analysis (IPDMA) [3] of four trials of HFOV vs. conventional ventilation in adults with ARDS. Because of limited availability of data regarding physiologic changes after randomization, the causal mediation analysis of hemodynamic change included data only from the OSCILLATE trial [4].

The main exposure was HFOV, and the primary outcome was all-cause 30-day mortality. The hypothesized effect modifier (risk of RV failure) was defined by the acute cor pulmonale (ACP) score measured at baseline (pre-randomization) [5]. The hypothesized mediator was

\footnotetext{
*Correspondence: neill.adhikari@utoronto.ca

${ }^{2}$ Interdepartmental Division of Critical Care Medicine, University of Toronto, Toronto, Canada
}

Full author information is available at the end of the article change in hemodynamics at $2 \mathrm{~h}$ post-randomization, defined as any decrease in either systolic or diastolic blood pressure or any increase in vasopressor dose.

To estimate the effect of HFOV across ACP risk categories, we used generalized estimating equations with hospital-level clustering, with covariates selected a priori based on a directed acyclic graph and including the interaction term between HFOV and ACP score $\geq 2$. We then performed a causal mediation analysis to determine if the detrimental effect of HFOV on mortality was mediated by change in hemodynamics (see Online appendix). All reported $p$ values are two-sided, with $p<0.05$ interpreted as statistically significant. Analyses were performed in STATA v.14.2.

Baseline patient characteristics are shown in the Online appendix. For each point increase in the ACP score, there was a $23 \%$ increase in the odds of hemodynamic worsening [odds ratio (OR) 1.23, 95\% confidence interval (CI) 1.02-1.47]. Patients with ACP score $\geq 2$ at baseline were more likely to die (OR 1.79, 95\% CI 1.26-2.53, Table 1). However, the effect of HFOV was not modified by the high ACP score (interaction $p=0.18$ ). Our results are reported across subgroups defined by $\mathrm{PaO}_{2} / \mathrm{FiO}_{2}$ ratio.

In the OSCILLATE trial $(n=548)$, the increased mortality among patients receiving HFOV was not mediated by $2 \mathrm{~h}$ hemodynamic changes (proportion mediated: $1.1 \%$, 95\% CI $0.0-13.4, p=0.56$, Table 1). These results were consistent using alternative definitions of hemodynamic change (cardiac output and MAP). 
Table 1 Modification of effect on 30-day mortality by baseline risk of right ventricle failure and mediation by hemodynamic changes after randomization among adult patients with ARDS in HFOV trials

\begin{tabular}{|c|c|c|}
\hline Effect modification & Adjusted OR $(95 \% \mathrm{Cl})^{\mathrm{a}}$ & $p$ value \\
\hline Acute cor pulmonale score ${ }^{b} \geq 2$ & $1.79(1.26-2.53)$ & $<0.01$ \\
\hline \multicolumn{3}{|l|}{ HFOV treatment } \\
\hline Overall & $1.23(0.96-1.57)$ & 0.10 \\
\hline \multicolumn{3}{|l|}{ Subgroup analysis ${ }^{c}$} \\
\hline Patients with $\mathrm{PaO}_{2} / \mathrm{FiO}_{2}<100$ & $0.94(0.66-1.35)$ & 0.75 \\
\hline Patients with $\mathrm{PaO}_{2} / \mathrm{FiO}_{2} \geq 100$ & $1.48(1.12-1.94)$ & $<0.01$ \\
\hline Causal mediation by hemodynamic change ${ }^{d}$ & Adjusted RR $(95 \% \mathrm{Cl})^{e}$ & $p$ value \\
\hline Natural direct effect & $1.35(1.12-1.68)$ & 0.03 \\
\hline Natural indirect effect & $1.00(0.99-1.03)^{f}$ & 0.81 \\
\hline
\end{tabular}

$A R D S$ acute respiratory distress syndrome, $\mathrm{Cl}$ confidence interval, $\mathrm{HFOV}$ high-frequency oscillatory ventilation, $\mathrm{OR}$ odds ratio, $\mathrm{RR}$ risk ratio, $\mathrm{RV}$ right ventricle, $\mathrm{PaO} / \mathrm{FiO} \mathrm{O}_{2}$ arterial oxygen partial pressure to fractional inspired oxygen ratio, NIE natural indirect effect, $T E$ total effect, $C D E$ controlled direct effect, $m$ mediator, $A P A C H E$ II Acute Physiology and Chronic Health Evaluation II

a Based on a multivariable generalized estimating equation with a logit link, a binomial distribution, clustering at the hospital level and including: age, age ${ }^{2}$, gender, RV score, HFO treatment allocation, APACHE II, APACHE II ${ }^{2}$, tidal volume, trial, $\mathrm{PaO}_{2} / \mathrm{FiO}_{2}$, the interaction between $\mathrm{PaO} / \mathrm{FiO}$ and $\mathrm{HFOV}$, and duration of mechanical ventilation prior to randomization. The interaction term between HFOV and RV score as a binary covariate was not statistically significant ( $p=0.18$ )

b The acute cor pulmonale (ACP) score includes pneumonia as a cause of $A R D S$, driving pressure $\geq 18 \mathrm{~cm}_{2} \mathrm{O}$, arterial oxygen partial pressure to fractional inspired oxygen $\left(\mathrm{PaO}_{2} / \mathrm{FiO}_{2}\right)$ ratio $<150 \mathrm{mmHg}$, and arterial carbon dioxide partial pressure $\geq 48 \mathrm{mmHg}$

c Threshold taken from Meade et.al (Fig. 1, reference 4) and is the value of $\mathrm{PaO}_{2} / \mathrm{FiO}_{2}$ where the estimated odds ratio of death for $\mathrm{HFOV}$ changes to $\leq 1$

d Hemodynamic change was assessed $2 \mathrm{~h}$ post-randomization and defined by any increased dose of vasopressor or any decrease in either systolic or diastolic blood pressure. The natural direct effect (NDE) expresses how much the outcome of 30-day mortality would change in HFOV vs. conventional mechanical ventilation if the mediator of hemodynamic change were kept at the level it would have taken under conventional ventilation. The natural indirect effect (NIE) expresses how much the outcome would change if the exposure were set to HFOV and the mediator were changed from the level it would take under conventional ventilation to the level it would take under HFOV. The total effect (TE) is the overall change in outcome from conventional ventilation to HFOV groups and is the sum of NDE and NIE

e Models for the outcome were fitted using log-link and bootstrapping-based confidence intervals. Models for the mediator were fitted with a logit link and a binomial distribution. Models allow for mediator-exposure interaction. All models assume no exposure-mediator, exposure-outcome and mediator-outcome confounding

f Proportion mediated (NIE/TE): $1.1 \%(0.0-13.4)$ and proportion eliminated [(TE $-\mathrm{CDE}(m=0)) / \mathrm{TE})]: 6.8 \%$ (0.0-26.4). Confidence intervals for proportion mediated and eliminated are truncated at $0 \%$

Our study shows that the baseline risk of RV failure among patients with ARDS does not modify the effect of HFOV on 30-day mortality. Furthermore, the effect of HFOV is not mediated by immediate hemodynamic changes. However, patients with a higher baseline ACP risk score are at increased risk of death, a finding likely explained by illness severity. Limitations of the study include lack of echocardiographic definition of RV failure at baseline and after randomization (see Online appendix). Further studies should evaluate specific subgroups of patients with very severe ARDS that may benefit from this treatment strategy.

\section{Electronic supplementary materia}

The online version of this article (https://doi.org/10.1007/s00134-019-05806-8) contains supplementary material, which is available to authorized users.

\footnotetext{
Author details

1 Department of Critical Care Medicine, Sunnybrook Health Sciences Centre, Toronto, Canada. ${ }^{2}$ Interdepartmental Division of Critical Care Medicine, University of Toronto, Toronto, Canada. ${ }^{3}$ Institute of Health Policy, Management and Evaluation, Dalla Lana School of Public Health, University of Toronto, Toronto, Canada. ${ }^{4}$ Department of Medicine, Sinai Health System
}

and University Health Network, Toronto, Canada. ${ }^{5}$ Research Institute, Toronto General Hospital, Toronto, Canada. ${ }^{6}$ Pediatric Intensive Care Unit, University Medical Center Utrecht, Utrecht, The Netherlands. ${ }^{7}$ Department of Biomedical Technology, School of Biomedical Engineering, Czech Technical University in Prague, Prague, Czech Republic. ${ }^{8}$ Department of Medicine, Université de Sherbrooke, Sherbrooke, Canada.

\section{Author contributions}

FA, BLF, and NKJA conceived of the study. BHC, NDF, CWB, and TEB contributed data from the original trials of HFOV. FA analysed the data. FA, BLF, and NKJA drafted the manuscript. All authors interpreted data, revised the manuscript, and approved the final version. NKJA supervised the study.

\section{Funding}

Dr. Angriman is partially supported by research funding from the Department of Critical Care Medicine, Sunnybrook Health Sciences Centre.

\section{Compliance with ethical standards}

Conflicts of interest

On behalf of all authors, the corresponding author states that there is no conflict of interest.

\section{Publisher's Note}

Springer Nature remains neutral with regard to jurisdictional claims in published maps and institutional affiliations. 
Accepted: 26 September 2019

Published online: 29 October 2019

\section{References}

1. Calfee CS, Delucchi K, Parsons PE et al (2014) Subphenotypes in acute respiratory distress syndrome: latent class analysis of data from two randomised controlled trials. Lancet Respir Med 2:611-620. https://doi. org/10.1016/S2213-2600(14)70097-9

2. Sklar MC, Fan E, Goligher EC (2017) High-frequency oscillatory ventilation in adults with ARDS. Chest 152:1306-1317. https://doi.org/10.1016/j. chest.2017.06.025
3. Meade MO, Young D, Hanna S et al (2017) Severity of hypoxemia and effect of high-frequency oscillatory ventilation in acute respiratory distress syndrome. Am J Respir Crit Care Med 196:727-733. https://doi. org/10.1164/rccm.201609-1938OC

4. Ferguson ND, Cook DJ, Guyatt GH et al (2013) High-frequency oscillation in early acute respiratory distress syndrome. N Engl J Med 368:795-805. https://doi.org/10.1056/NEJMoa1215554

5. Mekontso Dessap A, Boissier F, Charron C et al (2016) Acute cor pulmonale during protective ventilation for acute respiratory distress syndrome: prevalence, predictors, and clinical impact. Intensive Care Med 42:862-870. https://doi.org/10.1007/s00134-015-4141-2 